\title{
Handbuch Hochschulbibliothekssysteme: leistungsfähige Infor-
}

mationsinfrastrukturen für Wissenschaft und Forschung, hrsg. von Konstanze Söllner und Wilfried Sühl-Stromenger [unter Mitarb. von Martina Straub], Berlin: De Gruyter Saur, 2014, 608 S. ISBN 978-3-11-030991-1 e-ISBN (PDF) 978-3-11-031009-2
e-ISBN (EPUB) 978-3-11-039621-8

Bei der Lektüre dieses Buches wird man - zumindest als Komparatistin - gleich zu Beginn von dem vorangestellten Motto von Walt Whitmans „Crossing Brooklyn Ferry" in den Bann gezogen. Dieses Werk, aus der Mitte des 19. Jahrhunderts, gewidmet den Fährfahrten, stellt die Stadt als Thema und das Leben in der Masse ins Zentrum, die Stadt wird zum Inbegriff des modernen Lebens. Whitman gilt als Begründer der modernen amerikanischen Dichtung und sein grosser Einfluss auf die Lyrik des 19. Jahrhundert - aber nicht nur darauf - ist unbestritten.

Nun, die Frage ist, was hat Walt Whitman mit den Hochschulbibliothekssystemen gemein? Mal sehen, ob sich diese Frage beantworten lässt.

Beim Durchsehen des Inhaltsverzeichnisses fällt auf, dass die Themen ein sehr breites Spektrum aufweisen. Neben Planungsempfehlungen des Wissenschaftsrats und der DFG werden Bibliothekskonzepte aus den diversen Wissenschaftsdisziplinen vorgestellt: von „Hochschulpolitik, Hochschulentwicklung und Hochschulrecht - Vorgaben für die Ausgestaltung von Bibliothekssystemen“ über „Bibliotheksstrukturen im Spannungsfeld von Zentralität und Dezentralität" aber eben auch bis hin zum Wandel der Bibliotheken in den unterschiedlichen Wissenschaftsdisziplinen, Kooperationen von Organisationseinheiten und/oder Institutionen innerhalb und ausserhalb einer Hochschule und zentralen Koordinationseinrichtungen für Bibliotheken bzw. deren Hochschulen. Die einzelnen Beiträge ergeben noch ein breiteres Bild der und einen tieferen Einblick in die aktuellen Herausforderungen von Universitätsbibliotheken: es werden geschichtliche Entwicklungen verschiedener Universitätsbibliotheken aufgezeigt (zum Bei- 
spiel Wilfried Lochbühlers „Dreischichtigkeit zwischen Diversifikation und Kooperation" der Universitätsbibliothek Zürich oder jener der UB Wien von Maria Seissl und Wolfgang Nikolaus Rappert „Das Bibliothekssystem an der Universität Wien“). Die letzten Jahrzehnte sind stark geprägt von Veränderungen und Abhängigkeiten aufgrund von Reorganisationen der Verwaltungsstrukturen und Zusammenlegungen bzw. neuen Standorten bis hin zu Campusbildungen (beispielsweise an der UB Basel) sowie neuen Funktionen und Aufgabenfeldern. Der Fokus der meisten Beiträge mit geschichtlichen Abrissen liegt auf den letzten Jahren, gleichzeitig gewähren die Aufsätze auch einen spannenden Ausblick auf die kommenden Jahre, die Themen reichen von „Lernorte“ (zum Beispiel „Die Campus-Bibliothek Bergheim an der Universität Heidelberg“ von Marion Krüger) bis Innovationsmanagement (beispielsweise Rudolf Mummenthalers „Innovationsmanagement und Steuerung des Reformprozesses" oder der Beitrag der UB Wien).

Ebenso finden sich in dem Handbuch interessante Beiträge zu Discovery Services („Das Wissensportal der Bibliothek der ETH Zürich“ von Wolfram Neubauer und Arlette Piguet), Mitarbeitendenentwicklung („Personalführung in grossen Bibliothekssystemen" von Beate Tröger) und Leistungsmessung und Benchmarking im Bibliothekswesen („Exzellenz und Mittelmass" von Bruno Bauer und Robert Schiller), dies ergibt einen weit grösseren Bogen als das Thema Bibliothekssysteme vorgibt.

Viele der Beiträge drehen sich um die zentrale Frage der Ein- oder Mehrschichtigkeit, wie der Artikel von Bettina Koeper der Universitätsbibliothek Bielefeld: „Strukturvorteile durch Einschichtigkeit?" Mit diesem Satz beantwortet sie m.E. die im Titel des Beitrages gestellte Frage aus der Aussensicht, und zwar jener der Universität als Trägerin und Leistungsbezieherin der bibliothekarischen Services: „Letztlich erweist sich die Einschichtigkeit aber auch für die Universität als vorteilhaft, da sie mit der Bibliothek einen dezidierten Ansprechpartner hat und sich dadurch hochschulübergreifende Entwicklungen leichter initialisieren lassen als beispielsweise in zweischichtigen Systemen..." (S. 101, Koeper Bettina: S. 94-102). Damit wird klar, dass die Hochschulbibliotheken keine "stand-alone-Lösungen“ mehr darstellen und nicht mehr eigenständig und losgelöst vom universitären Betrieb sind. Ihre Existenzen ergeben sich auch nicht mehr aus der Jahrhunderte oder gar Jahrtausende alten Tradition und Aufgabe heraus, sondern sie sind im Idealfall völlig integriert und angewiesen auf eine gute Zusammenarbeit mit den diversen Anspruchsgruppen um auch für die Zukunft die Dienstleistungseinrichtung für die universitäre Informationsversorgung zu sein. 
Nun, die anfangs gestellte Frage: was hat es im Rahmen von Hochschulbibliothekssystemen mit Walt Whitmans Versen auf sich?

Die Metapher von der verbindenden Fähre passt vielleicht zu den dezentral organisierten Bibliothekssystemen, in denen es auf Verbindungen, Vernetzungen, Überbrückungen ankommt. Sicherlich gilt diese Metapher aber für Bibliotheken: sie stellen Verbindungen zwischen Informationsanbietern und Informationssuchenden her.

In diesem Sinne bietet dieses Buch eine Fülle an Best-Practice-Beispielen aus den unterschiedlichsten Aufgabengebieten im Bibliotheksmanagement und ist eine Fundgrube für all jene, die Anregungen und Ideen für die eigene Bibliotheksorganisation suchen.

Edeltraud Haas, St. Gallen

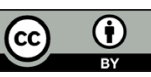

Dieses Werk ist lizenziert unter einer Creative-Commons-Lizenz Namensnennung 4.0 International 